\title{
Associations between coffee consumption and all-cause and cause-specific mortality in a Japanese city: the Takayama study
}

\author{
Michiyo Yamakawa ${ }^{1, *}$, Keiko Wada ${ }^{1}$, Yuko Goto ${ }^{1}$, Fumi Mizuta ${ }^{1,2}$, Sachi Koda ${ }^{1,3}$, \\ Takahiro Uji ${ }^{1}$ and Chisato Nagata ${ }^{1}$ \\ 'Department of Epidemiology and Preventive Medicine, Gifu University Graduate School of Medicine, 1-1 Yanagido, \\ Gifu 501-1 194, Japan: ${ }^{2}$ Department of Nutrition, Shubun University, Aichi, Japan: ${ }^{3}$ Department of Human Nutrition, \\ Sugiyama Jogakuen University, Aichi, Japan
}

Submitted 1 November 2018: Final revision received 13 February 2019: Accepted 14 February 2019: First published online 20 May 2019

\begin{abstract}
Objective: Epidemiological studies suggest that coffee consumption is inversely associated with all-cause and cause-specific mortality. Evidence from studies targeting non-white, non-Western populations is still sparse, although coffee is popular and widely consumed in Asian countries.

Design: Population-based, prospective cohort study. We used Cox proportional hazards models with adjustment for dietary and lifestyle factors to estimate associations between coffee consumption and all-cause and cause-specific mortality. Dietary intake including coffee consumption was assessed only at baseline using a validated FFQ.

Setting: A Japanese city.

Participants: Individuals aged 35 years or older without cancer, CHD and stroke at baseline ( $n$ 29 079) and followed from 1992 to 2008.

Results: From 410352 person-years, 5339 deaths were identified (mean followup $=14 \cdot 1$ years). Coffee consumption was inversely associated with mortality from all causes and CVD among all participants, but not from cancer. Compared with the category of 'none', the multivariate hazard ratio (95\% CI) for all-cause mortality was $0.93(0.86,1.00)$ for $<1 \mathrm{cup} / \mathrm{d}, 0.84(0.76,0.93)$ for $1 \mathrm{cup} / \mathrm{d}$ and $0.81(0.71$, $0.92)$ for $2-3 \mathrm{cups} / \mathrm{d}$. The multivariate hazard ratio $(95 \% \mathrm{CI})$ for cardiovascular mortality were $0.87(0.77,0.99)$ for $<1 \mathrm{cup} / \mathrm{d}, 0.76(0.63,0.92)$ for $1 \mathrm{cup} / \mathrm{d}$ and $0.67(0.50,0.89)$ for $2-3 \mathrm{cups} / \mathrm{d}$. Inverse associations were also observed for mortality from other causes, specifically infectious and digestive diseases.

Conclusion: Drinking coffee, even $1 \mathrm{cup} / \mathrm{d}$, was inversely associated with all-cause mortality and mortality from cardiovascular, infectious and digestive diseases.
\end{abstract}

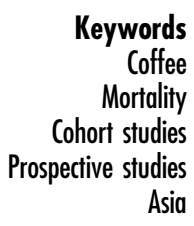

Coffee may have a substantial impact on health because of its many bioactive compounds with potential therapeutic antioxidant, anti-inflammatory, antifibrotic or anticancer effects (e.g. the alkaloids, caffeine and trigonelline; chlorogenic acids; and the diterpenes, cafestol and kahweol $)^{(1-3)}$. Four meta-analyses showed that coffee consumption was inversely associated with all-cause and CVD mortality ${ }^{(4-7)}$. Furthermore, a recent review of meta-analyses suggested that coffee consumption was more often associated with benefit than harm for a range of health outcomes, including all-cause and CVD mortality ${ }^{(8)}$. The evaluation was performed on existing studies, which were mainly from Western countries. However, evidence from studies targeting non-white, non-Western populations is still sparse, although lifestyles and disease risks can vary by ethnicity $^{(9,10)}$.
Recent studies targeting multi-ethnic, multinational populations reported that coffee consumption was inversely associated with all-cause mortality and such associations did not vary by ethnicity or country ${ }^{(11,12)}$. In two meta-analyses $^{(4,6)}$ (including three Japanese cohort studies $\left.{ }^{(13-15)}\right)$, coffee consumption was also inversely associated with all-cause mortality. A subsequent cohort study in Japan reported that coffee consumption was inversely associated with mortality from all causes, CVD and respiratory disease ${ }^{(16)}$. Coffee is popular and widely consumed in Asian countries, although the consumption seems to be lower than that in Western countries ${ }^{(17)}$. Past studies in Singapore and Korea reported that a little coffee consumption was inversely associated with hypertension and metabolic syndrome ${ }^{(18,19)}$. Thus, studying coffee use among Japanese community-dwelling people will 
aid in providing further insights into its associations with health.

In the present study, we examined the associations between coffee consumption and all-cause and cause-specific mortality with adjustment for important dietary and lifestyle factors using data from a population-based cohort in a Japanese city. We evaluated the health effects of fewer cups of coffee consumption than that in Western countries because the average consumption is lower in Japan (1-2 cups/person per d).

\section{Methods}

\section{Study participants}

The Takayama cohort study was initiated in 1992 and targeted all residents aged 35 years or older in Takayama city, Gifu Prefecture, Japan, to examine lifestyle and dietary factors associated with health. The study details were described previously ${ }^{(20)}$. In total, 31552 residents (85.3\%) completed a self-administered questionnaire at baseline, including questions on demographic characteristics, body weight and height, smoking, alcohol drinking, diet and physical activities. We excluded participants who reported a prior diagnosis of cancer, CHD and stroke at baseline ( $n$ 2473), which left 29079 participants (13355 men and 15724 women) in the analyses.

\section{Follow-up and end point}

The participants were followed from the baseline survey in 1992 to the date of death or the end of follow-up on 1 October 2008. The data on participants who died or moved out of Takayama city were obtained from basic resident or family registration databases. We identified the underlying causes of death using death certificates provided by the Legal Affairs Bureau, which were coded according to the International Classification of Disease, Tenth Revision (ICD-10). The main end points were all-cause mortality and mortality due to cancer (ICD-10: C00-D48), CVD (ICD-10: I00-I99; IHD: I20-I25; stroke: I60-I69) and other causes. Furthermore, respiratory disease (ICD-10: J10-J18 and J40-J47), injury (ICD-10: S00-T98), digestive disease (ICD-10: K00-K93) and infectious disease (ICD-10: A00 -B99) were selected as additional end points.

\section{Coffee consumption}

Dietary intake including coffee consumption was assessed only at baseline by a validated 169-item semi-quantitative FFQ. The participants were asked to report how often and what amount they consumed for each food and beverage item during the past year. Nutrient intakes were estimated from the information on frequency and portion size using the fifth revised and enlarged edition of the Japanese Standard Tables of Food Composition. Details of the FFQ and the methods used for calculating nutrient intakes were described previously ${ }^{(21,22)}$. The frequency of coffee consumption during the past year was determined from the questionnaire at baseline and was originally grouped into nine categories: 'none', 'once per month', '2-3 times per month', 'once per week', '2-3 times per week', '4-6 times per week', 'once per day', '2-3 times per day' and ' $\geq 4$ times per day'. Participants who drank coffee every day were presumed to answer with the number of coffees they drank in a day. We classified participants who drank coffee occasionally ('once per month', '2-3 times per month', 'once per week', '2-3 times per week' and '4-6 times per week') into a single category $(<1 \mathrm{cup} / \mathrm{d})$. Finally, the participants were categorized as follows: none, $<1 \mathrm{cup} / \mathrm{d}, 1 \mathrm{cup} / \mathrm{d}, 2-3 \mathrm{cups} / \mathrm{d}$ and $\geq 4$ cups/d. We utilized $12 \mathrm{~d}$ dietary records over a year to validate the estimated coffee consumption from the questionnaire. Spearman's correlation coefficients of estimated frequency (the number of cups) between the questionnaire and the records were 0.73 in men and 0.58 in women. We extrapolated the median frequency of coffee consumption using the results from all participants into the data for participants with missing information.

\section{Covariates}

We considered the following variables measured at baseline as potential a priori confounders: age; sex; marital status (married or not married (single, divorced/separated or widowed)); years of education ( $\leq 8,9-11,12-14$ or $\geq 15$ years); $\mathrm{BMI}$ ( $<18.5,18.5-24.9, \geq 25.0 \mathrm{~kg} / \mathrm{m}^{2}$ or missing); history of diabetes (no or yes); smoking status (never, former or current); alcohol intake (continuous); weekly vigorous exercise (never, 1, 2, 3, 4, 5, 6 or $\geq 7$ times); use of any vitamin supplement (no or yes); total daily energy intake (continuous); daily intakes of vegetables and fruits, and red meat (continuous); and consumption of black tea, green tea, Chinese tea and soda (none, $<1$ or $\geq 1$ times/d). Alcohol intake was categorized into five categories: nondrinkers or drinkers $(<2.9,2.9-<15.8,15 \cdot 8-<44.7$ or $\geq 44.7 \mathrm{mg} / \mathrm{d}$ ). In the stratified analyses by sex, alcohol intake was categorized into quartiles for men and into three categories for women (i.e. non-drinkers, or drinkers below or above the median for alcohol consumption). Daily intakes of vegetables and fruits, and red meat were adjusted for total energy intake using the residual method of energy adjustment $^{(23)}$.

\section{Statistical analysis}

Person-years for each study participant were counted from the date of the baseline survey to the date of death, date of censorship or the end of follow-up (1 October 2008), whichever occurred first. Using Cox proportional hazards models, we estimated age- and sex-adjusted hazard ratios (HR) and $95 \%$ CI for associations of coffee consumption with all-cause and cause-specific mortality using the 'none' 
Table 1 Baseline characteristics of total participants according to daily coffee consumption: Japanese adults aged $\geq 35$ years ( $n 29079$ ) followed from 1992 to 2008, Takayama study

\begin{tabular}{|c|c|c|c|c|c|c|c|c|c|c|}
\hline & \multicolumn{10}{|c|}{ Daily coffee consumption } \\
\hline & \multicolumn{2}{|c|}{ None $(n$ 6792) } & \multicolumn{2}{|c|}{$<1$ cup $(n 11338)$} & \multicolumn{2}{|c|}{1 cup $(n 6088)$} & \multicolumn{2}{|c|}{$2-3$ cups $(n 4378)$} & \multicolumn{2}{|c|}{$\geq 4$ cups $(n 483)$} \\
\hline & $\begin{array}{l}\text { Mean, } n \\
\text { or median }\end{array}$ & $\begin{array}{l}\text { SD, \% or } \\
\text { IQR }\end{array}$ & $\begin{array}{l}\text { Mean, } n \\
\text { or median }\end{array}$ & $\begin{array}{l}\text { SD, \% or } \\
\text { IQR }\end{array}$ & $\begin{array}{l}\text { Mean, } n \\
\text { or median }\end{array}$ & $\begin{array}{l}\text { SD, \% or } \\
\text { IQR }\end{array}$ & $\begin{array}{l}\text { Mean, } n \\
\text { or median }\end{array}$ & $\begin{array}{l}\text { SD, \% or } \\
\text { IQR }\end{array}$ & $\begin{array}{l}\text { Mean, } n \\
\text { or median }\end{array}$ & $\begin{array}{l}\text { SD, \% or } \\
\text { IQR }\end{array}$ \\
\hline Age (years), mean and SD & $63 \cdot 6$ & $12 \cdot 6$ & $55 \cdot 0$ & $11 \cdot 7$ & $50 \cdot 4$ & $10 \cdot 4$ & $46 \cdot 4$ & 8.6 & $45 \cdot 2$ & $7 \cdot 6$ \\
\hline $\begin{array}{l}\text { Men, } n \text { and } \% \\
\text { Marital status, } n \text { and \% }\end{array}$ & 2645 & \multicolumn{8}{|c|}{ Marital status, $n$ and $\%$} & $56 \cdot 5$ \\
\hline Unmarried & 1833 & $27 \cdot 6$ & 1746 & $15 \cdot 6$ & 802 & $13 \cdot 3$ & 516 & 11.9 & 74 & $15 \cdot 5$ \\
\hline Married & 4797 & $72 \cdot 4$ & 9474 & $84 \cdot 4$ & 5218 & $86 \cdot 7$ & 3831 & $88 \cdot 1$ & 404 & 84.5 \\
\hline \multicolumn{11}{|l|}{ Education years, $n$ and $\%$} \\
\hline$\leq 8$ years & 3286 & $49 \cdot 6$ & 2629 & 23.5 & 779 & $12 \cdot 9$ & 257 & 5.9 & 20 & $4 \cdot 2$ \\
\hline$\overline{9}-11$ years & 2093 & 31.6 & 4476 & 39.9 & 2504 & 41.5 & 1572 & $36 \cdot 3$ & 162 & 34.0 \\
\hline $12-14$ years & 1016 & $15 \cdot 3$ & 3307 & 29.5 & 2154 & $35 \cdot 7$ & 1911 & $44 \cdot 2$ & 217 & $45 \cdot 6$ \\
\hline$\geq 15$ years & 230 & 3.5 & 796 & $7 \cdot 1$ & 598 & 9.9 & 587 & $13 \cdot 6$ & 77 & $16 \cdot 2$ \\
\hline $\begin{array}{l}\mathrm{BMI}\left(\mathrm{kg} / \mathrm{m}^{2}\right), \text { mean and SD } \\
\text { Diabetes, } n \text { and } \%\end{array}$ & \multicolumn{9}{|c|}{ Diabetes, $n$ and $\%$} & $2 \cdot 8$ \\
\hline No & 6443 & 94.9 & 10892 & $96 \cdot 1$ & 5924 & $97 \cdot 3$ & 4281 & $97 \cdot 8$ & 470 & $97 \cdot 3$ \\
\hline Yes & 349 & $5 \cdot 1$ & 446 & 3.9 & 164 & $2 \cdot 7$ & 97 & $2 \cdot 2$ & 13 & $2 \cdot 7$ \\
\hline \multicolumn{11}{|l|}{ Smoking status, $n$ and $\%$} \\
\hline Never & 3520 & $51 \cdot 8$ & 5915 & $52 \cdot 2$ & 2810 & $46 \cdot 2$ & 1459 & $33 \cdot 3$ & 98 & $20 \cdot 3$ \\
\hline Former & 1102 & $16 \cdot 2$ & 1855 & $16 \cdot 4$ & 808 & $13 \cdot 3$ & 477 & $10 \cdot 9$ & 31 & 6.4 \\
\hline Current & 1292 & $19 \cdot 0$ & 2861 & $25 \cdot 2$ & 2178 & $35 \cdot 8$ & 2315 & $52 \cdot 9$ & 338 & $70 \cdot 0$ \\
\hline Missing & 878 & $12 \cdot 9$ & 707 & $6 \cdot 2$ & 292 & 4.8 & 127 & 2.9 & 16 & 3.3 \\
\hline $\begin{array}{l}\text { Alcohol intake }(\mathrm{mg} / \mathrm{d}) \\
\text { median and IQR }\end{array}$ & 1.9 & $0-27 \cdot 9$ & $6 \cdot 3$ & $0.9-34.7$ & $8 \cdot 1$ & $1 \cdot 1-37 \cdot 8$ & $12 \cdot 5$ & $1.5-40 \cdot 6$ & $8 \cdot 3$ & $0.9-36 \cdot 7$ \\
\hline $\begin{array}{l}\text { Vigorous exercise } \geq 5 \text { times/ } \\
\text { week, } n \text { and } \%\end{array}$ & 1089 & $17 \cdot 9$ & 2042 & $18 \cdot 8$ & 1111 & $19 \cdot 0$ & 771 & $18 \cdot 0$ & 105 & $22 \cdot 1$ \\
\hline \multicolumn{11}{|l|}{$\begin{array}{l}\text { Use of any vitamin } \\
\text { supplement, } n \text { and \% }\end{array}$} \\
\hline No & 3945 & $58 \cdot 1$ & 7311 & 64.5 & 4024 & $66 \cdot 1$ & 3107 & $71 \cdot 0$ & 344 & $71 \cdot 2$ \\
\hline Yes & 2030 & 29.9 & 3500 & $30 \cdot 9$ & 1765 & $29 \cdot 0$ & 1155 & $26 \cdot 4$ & 128 & 26.5 \\
\hline Missing & 817 & $12 \cdot 0$ & 527 & 4.7 & 299 & 4.9 & 116 & $2 \cdot 7$ & 11 & $2 \cdot 3$ \\
\hline \multicolumn{11}{|l|}{ Dietary intake, mean and SD } \\
\hline Total energy (kJ/d) & $8796 \cdot 4$ & $3435 \cdot 9$ & $9854 \cdot 2$ & $3458 \cdot 1$ & 10287.6 & $3587 \cdot 8$ & $10782 \cdot 6$ & $3773 \cdot 1$ & $11332 \cdot 8$ & $4301 \cdot 2$ \\
\hline Total energy (kcal/d) & $2102 \cdot 4$ & $821 \cdot 2$ & $2355 \cdot 2$ & $826 \cdot 5$ & 2458.8 & 857.5 & $2577 \cdot 1$ & $901 \cdot 8$ & $2708 \cdot 6$ & 1028.0 \\
\hline Vegetables and fruits $(\mathrm{g} / \mathrm{d})$ & $550 \cdot 7$ & 273.3 & $512 \cdot 3$ & 259.4 & $499 \cdot 1$ & $273 \cdot 2$ & 453.9 & 263.6 & $453 \cdot 7$ & 269.5 \\
\hline Red meat $(g / d)$ & 31.5 & 20.5 & 35.4 & 21.8 & $37 \cdot 1$ & $23 \cdot 6$ & $37 \cdot 8$ & $25 \cdot 2$ & $40 \cdot 9$ & 31.0 \\
\hline \multicolumn{11}{|l|}{$\begin{array}{l}\text { Beverage consumption, } n \\
\text { and } \% \\
\text { Black tea }\end{array}$} \\
\hline None & 5798 & 85.4 & 7082 & 62.5 & 4012 & 65.9 & 2839 & 64.9 & 326 & 67.5 \\
\hline$<1$ time/d & 885 & $13 \cdot 0$ & 4075 & 35.9 & 1925 & 31.6 & 1459 & $33 \cdot 3$ & 142 & 29.4 \\
\hline$\geq 1$ time/d & 109 & 1.6 & 181 & 1.6 & 151 & $2 \cdot 5$ & 80 & $1 \cdot 8$ & 15 & $3 \cdot 1$ \\
\hline $\begin{array}{l}\text { Green tea } \\
\text { None }\end{array}$ & 2444 & $36 \cdot 0$ & 3109 & $27 \cdot 4$ & 1616 & $26 \cdot 5$ & 1186 & $27 \cdot 1$ & 128 & 26.5 \\
\hline$<1$ time/d & 604 & 8.9 & 1825 & $16 \cdot 1$ & 839 & $13 \cdot 8$ & 738 & $16 \cdot 9$ & 104 & 21.5 \\
\hline $\begin{array}{l}\geq 1 \text { time } / \mathrm{d} \\
\text { Chinese tea }\end{array}$ & 3744 & Chinese tea & 6404 & $56 \cdot 5$ & 3633 & $59 \cdot 7$ & 2454 & $56 \cdot 1$ & 251 & $52 \cdot 0$ \\
\hline None & 4215 & $62 \cdot 1$ & 4149 & $36 \cdot 6$ & 2304 & $37 \cdot 8$ & 1429 & $32 \cdot 6$ & 166 & 34.4 \\
\hline$<1$ time/d & 1892 & $27 \cdot 9$ & 5883 & 51.9 & 2773 & $45 \cdot 6$ & 2123 & 48.5 & 230 & $47 \cdot 6$ \\
\hline$\geq 1$ time $/ \mathrm{d}$ & 685 & $10 \cdot 1$ & 1306 & 11.5 & 1011 & $16 \cdot 6$ & 826 & 18.9 & 87 & $18 \cdot 0$ \\
\hline \multicolumn{11}{|l|}{ Soda } \\
\hline None & 4257 & $62 \cdot 7$ & 4002 & $35 \cdot 3$ & 2374 & 39.0 & 1471 & $33 \cdot 6$ & 165 & $34 \cdot 2$ \\
\hline$<1$ time $/ \mathrm{d}$ & 2387 & $35 \cdot 1$ & 7077 & $62 \cdot 4$ & 3442 & $56 \cdot 5$ & 2619 & $59 \cdot 8$ & 281 & $58 \cdot 2$ \\
\hline$\geq 1$ time $/ \mathrm{d}$ & 148 & $2 \cdot 2$ & 259 & $2 \cdot 3$ & 272 & 4.5 & 288 & $6 \cdot 6$ & 37 & $7 \cdot 7$ \\
\hline
\end{tabular}

IQR, interquartile range.

category as a reference. Tests for linear trends in the associations were performed by assigning scores for the coffeeconsumption categories, which started from 0 for the lowest category (none) to 4 for the highest category ( $\geq 4$ cups/d). We then estimated multivariate HR after adjusting for all relevant confounding variables as mentioned above. We also conducted these analyses after stratifying by sex. In the analyses for cause-specific mortality (i.e. respiratory disease, injury, digestive disease and infectious disease), we collapsed the two higher categories into one category because of a small number of deaths in the category of $\geq 4$ cups/d. 
Table 2 Hazard ratios (HR) for mortality from all causes, cancer, CVD and other causes among total participants and participants stratified by sex, according to daily coffee consumption: Japanese adults aged $\geq 35$ years ( $n 29079$ ) followed from 1992 to 2008 , Takayama study

\begin{tabular}{|c|c|c|c|c|c|c|c|c|c|c|}
\hline & \multicolumn{9}{|c|}{ Daily coffee consumption } & \multirow[b]{3}{*}{$P$ for trend } \\
\hline & \multirow[b]{2}{*}{ None } & \multicolumn{2}{|r|}{$<1$ cup } & \multicolumn{2}{|r|}{1 cup } & \multicolumn{2}{|c|}{$2-3$ cups } & \multicolumn{2}{|c|}{$\geq 4$ cups } & \\
\hline & & $\mathrm{HR}$ & $95 \% \mathrm{Cl}$ & HR & $95 \% \mathrm{Cl}$ & $\mathrm{HR}$ & $95 \% \mathrm{Cl}$ & $\mathrm{HR}$ & $95 \% \mathrm{Cl}$ & \\
\hline \multicolumn{11}{|l|}{ Total } \\
\hline $\begin{array}{l}\text { Person-years } \\
\text { All-cause mortality }\end{array}$ & 88320 & \multicolumn{2}{|r|}{161424} & \multicolumn{2}{|c|}{89329} & \multicolumn{2}{|c|}{64298} & \multicolumn{2}{|r|}{6981} & \\
\hline Number of deaths & 2346 & \multicolumn{2}{|r|}{1964} & \multicolumn{2}{|r|}{652} & \multicolumn{2}{|r|}{334} & & 43 & \\
\hline Age- and sex-adjusted & 1.00 (ref.) & 0.86 & $0.81,0.91$ & 0.83 & $0.75,0.91$ & 0.92 & $0.81,1.03$ & 1.36 & $1.00,1.85$ & 0.003 \\
\hline Multivariate ${ }^{\star}$ & 1.00 (ref.) & 0.93 & $0.86,1.00$ & 0.84 & $0.76,0.93$ & 0.81 & $0.71,0.92$ & 1.09 & $0.80,1.50$ & $<0.001$ \\
\hline Cancer mortality & & & & & & & & & & \\
\hline Number of deaths & 568 & & 646 & & 237 & & 154 & & 15 & \\
\hline Age- and sex-adjusted & 1.00 (ref.) & 0.98 & $0.87,1.10$ & 0.91 & $0.78,1.07$ & 1.09 & $0.90,1.32$ & $1 \cdot 13$ & $0.67,1.90$ & 0.840 \\
\hline Multivariate* & 1.00 (ref.) & 1.03 & $0.90,1.17$ & 0.90 & $0.76,1.07$ & 0.99 & $0.80,1.22$ & 1.02 & $0.60,1.73$ & 0.541 \\
\hline CVD mortality & & & & & & & & & & \\
\hline Number of deaths & 851 & & 575 & & 174 & & 66 & & 12 & \\
\hline Age- and sex-adjusted & 1.00 (ref.) & 0.80 & $0.72,0.89$ & 0.77 & $0.65,0.91$ & 0.72 & $0.56,0.94$ & 1.60 & $0.90,2.84$ & $<0.001$ \\
\hline Multivariate* & 1.00 (ref.) & 0.87 & $0.77,0.99$ & 0.76 & $0.63,0.92$ & 0.67 & $0.50,0.89$ & 1.39 & $0.77,2.49$ & 0.002 \\
\hline Other cause mortality & & & & & & & & & & \\
\hline Number of deaths & 926 & 742 & & 241 & & 27 & & 5 & & \\
\hline Age- and sex-adjusted & 1.00 (ref.) & 0.85 & $0.77,0.94$ & 0.82 & $0.71,0.95$ & 0.86 & $0.70,1.06$ & 1.46 & $0.89,2.41$ & 0.021 \\
\hline Multivariate* & 1.00 (ref.) & 0.91 & $0.81,1.03$ & 0.85 & $0.73,1.00$ & 0.72 & $0.57,0.90$ & 1.00 & $0.58,1.72$ & 0.005 \\
\hline Men & & & & & & & & & & \\
\hline Person-years & 33043 & & 71023 & & 40581 & & 4750 & & 3903 & \\
\hline All-cause mortality & & & & & & & & & & \\
\hline Number of deaths & 1049 & & 1162 & & 417 & & 242 & & 31 & \\
\hline Age-adjusted & 1.00 (ref.) & 0.85 & $0.78,0.93$ & 0.80 & $0.71,0.90$ & 0.82 & $0.71,0.95$ & 1.23 & $0.86,1.77$ & 0.002 \\
\hline Multivariate* & 1.00 (ref.) & 0.88 & $0.80,0.97$ & 0.77 & $0.67,0.87$ & 0.70 & $0.59,0.82$ & 0.99 & $0.68,1.43$ & $<0.001$ \\
\hline Cancer mortality & & & & & & & & & & \\
\hline Number of deaths & 309 & & 400 & & 146 & & 108 & & 11 & \\
\hline Age-adjusted & 1.00 (ref.) & 0.93 & $0.80,1.08$ & 0.84 & $0.69,1.03$ & 1.02 & $0.81,1.29$ & $1 \cdot 15$ & $0.62,2.11$ & 0.694 \\
\hline Multivariate ${ }^{\star}$ & 1.00 (ref.) & 0.95 & $0.80,1.12$ & 0.79 & $0.63,0.98$ & 0.88 & $0.69,1.14$ & 1.00 & $0.54,1.86$ & $0 \cdot 120$ \\
\hline CVD mortality & & & & & & & & & & \\
\hline Number of deaths & 315 & & 303 & & 101 & & 47 & & 9 & \\
\hline Age-adjusted & 1.00 (ref.) & 0.77 & $0.66,0.91$ & 0.70 & $0.56,0.89$ & 0.61 & $0.44,0.84$ & 1.41 & $0.72,2.77$ & 0.001 \\
\hline Multivariate* & 1.00 (ref.) & 0.80 & $0.66,0.96$ & 0.68 & $0.53,0.87$ & 0.54 & $0.38,0.76$ & 1.21 & $0.61,2.40$ & $<0.001$ \\
\hline Other cause mortality & & & & & & & & & & \\
\hline Number of deaths & 425 & & 458 & & 170 & & 86 & & 11 & \\
\hline Age-adjusted & 1.00 (ref.) & 0.85 & $0.74,0.97$ & 0.85 & $0.71,1.02$ & 0.79 & $0.62,1.00$ & 1.21 & $0.66,2.22$ & 0.046 \\
\hline Multivariate $^{\star}$ & 1.00 (ref.) & 0.88 & $0.75,1.02$ & 0.81 & $0.67,1.00$ & 0.63 & $0.48,0.83$ & 0.85 & $0.45,1.62$ & 0.001 \\
\hline Women & & & & & & & & & & \\
\hline Person-years & 55277 & & 90402 & & 48749 & & 9548 & & 3078 & \\
\hline All-cause mortality & & & & & & & & & & \\
\hline Number of deaths & 1297 & & 802 & & 235 & & 92 & & 12 & \\
\hline Age-adjusted & 1.00 (ref.) & 0.88 & $0.81,0.97$ & 0.88 & $0.76,1.02$ & $1 \cdot 18$ & $0.94,1.48$ & 1.63 & $0.92,2.90$ & 0.499 \\
\hline Multivariate* & 1.00 (ref.) & 0.98 & $0.88,1.09$ & 0.93 & $0.80,1.10$ & 1.04 & $0.80,1.33$ & 1.14 & $0 \cdot 62,2 \cdot 10$ & 0.928 \\
\hline Cancer mortality & & & & & & & & & & \\
\hline Number of deaths & 259 & & 246 & & 91 & & 46 & & 4 & \\
\hline Age-adjusted & 1.00 (ref.) & 1.01 & $0.84,1.21$ & 0.99 & $0.77,1.29$ & 1.23 & $0.87,1.73$ & $1 \cdot 10$ & $0.41,2.99$ & 0.476 \\
\hline Multivariate* & 1.00 (ref.) & 1.08 & $0.87,1.33$ & 1.06 & $0.80,1.40$ & 1.21 & $0.83,1.76$ & 1.03 & $0.37,2.84$ & 0.517 \\
\hline CVD mortality & & & & & & & & & & \\
\hline Number of deaths & 536 & & 272 & & 73 & & 19 & & 3 & \\
\hline Age-adjusted & 1.00 (ref.) & 0.85 & $0.73,0.98$ & 0.87 & $0.67,1.12$ & 0.97 & $0.60,1.56$ & 1.55 & $0.50,4.86$ & 0.162 \\
\hline Multivariate & 1.00 (ref.) & 0.95 & $0.80,1.14$ & 0.86 & $0.65,1.14$ & 0.91 & $0.54,1.53$ & 1.24 & $0.39,3.98$ & 0.419 \\
\hline Other cause mortality & & & & & & & & & & \\
\hline Number of deaths & 501 & & 284 & & 71 & & 27 & & 5 & \\
\hline Age-adjusted & 1.00 (ref.) & 0.87 & $0.75,1.01$ & 0.78 & $0.60,1.01$ & 1.14 & $0.76,1.71$ & $2 \cdot 21$ & $0.91,5.38$ & 0.294 \\
\hline Multivariate* & 1.00 (ref.) & 0.96 & $0.80,1.15$ & 0.91 & $0.69,1.21$ & 0.91 & $0.56,1.47$ & 1.17 & $0.43,3.24$ & 0.742 \\
\hline
\end{tabular}

Ref., reference category.

*Marital status, years of education, BMI, diabetes, smoking status, alcohol intake, vigorous exercise, use of any vitamin supplement, dietary intake (total energy, vegetables and fruits, red meat) and beverage consumption (black tea, green tea, Chinese tea, soda) were also adjusted for.

In sensitivity analyses, we first estimated multivariate HR for all-cause mortality after excluding deaths within the first 3 years. To examine the impact of residual confounding from smoking status, we next conducted further analyses adjusting for smoking-years category (never, smoking
$<10,11-20,21-30, \geq 30$ years or smoking-years missing) instead of the smoking status category in the multivariate models. We also conducted the same analyses with smoking-cessation years category (currently smoking, <5, 6-10, $>10$ years of smoking cessation or never smoking). 
Table 3 Hazard ratios (HR) for mortality from other specific causes among total participants, according to daily coffee consumption: Japanese adults aged $\geq 35$ years ( $n$ 29 079) followed from 1992 to 2008, Takayama study

\begin{tabular}{|c|c|c|c|c|c|c|c|c|}
\hline & \multicolumn{7}{|c|}{ Daily coffee consumption } & \multirow[b]{3}{*}{$P$ for trenc } \\
\hline & \multirow[b]{2}{*}{ None } & \multicolumn{2}{|c|}{$<1$ cup } & \multicolumn{2}{|c|}{1 cup } & \multicolumn{2}{|c|}{$\geq 2$ cups $^{*}$} & \\
\hline & & $\mathrm{HR}$ & $95 \% \mathrm{Cl}$ & $\mathrm{HR}$ & $95 \% \mathrm{Cl}$ & $\mathrm{HR}$ & $95 \% \mathrm{Cl}$ & \\
\hline \multicolumn{9}{|l|}{ Respiratory disease } \\
\hline Number of deaths $(n 531)$ & 261 & & 186 & & 52 & & 32 & \\
\hline Multivariate† & 1.00 (ref.) & 0.95 & $0.76,1 \cdot 20$ & 0.87 & $0.62,1.21$ & 0.94 & $0.61,1.46$ & 0.497 \\
\hline \multicolumn{9}{|l|}{ Injury } \\
\hline Number of deaths ( $n 370)$ & 129 & & 131 & & 70 & & 40 & \\
\hline Multivariate† & 1.00 (ref.) & 0.82 & $0.61,1.09$ & 0.96 & $0.68,1.36$ & 0.74 & $0.48,1.14$ & 0.384 \\
\hline \multicolumn{9}{|l|}{ Digestive disease } \\
\hline Number of deaths ( $n$ 163) & 79 & & 61 & & 14 & & 9 & \\
\hline Multivariate $†$ & 1.00 (ref.) & 0.73 & $0.49,1.09$ & 0.34 & $0.17,0.66$ & 0.31 & $0.13,0.71$ & 0.001 \\
\hline \multicolumn{9}{|l|}{ Infectious disease } \\
\hline Number of deaths ( $n$ 146) & 73 & & 53 & & 12 & & 8 & \\
\hline Multivariate† & 1.00 (ref.) & 0.60 & $0.38,0.93$ & 0.44 & $0.22,0.88$ & 0.52 & $0.23,1.19$ & 0.012 \\
\hline \multicolumn{9}{|l|}{ Urologic disease } \\
\hline Number of deaths ( $n$ 112) & 53 & & 45 & & 12 & & 2 & \\
\hline Multivariate $\dagger$ & 1.00 (ref.) & $1 \cdot 33$ & $0 \cdot 80,2 \cdot 21$ & 1.04 & $0.50,2 \cdot 17$ & 0.40 & $0.09,1.78$ & 0.540 \\
\hline \multicolumn{9}{|l|}{ Endocrine metabolic disease } \\
\hline Number of deaths ( $n 79$ ) & 40 & & 22 & & 11 & & 6 & \\
\hline Multivariate $†$ & 1.00 (ref.) & 0.58 & $0.32,1.08$ & 0.71 & $0.33,1.54$ & 0.53 & $0.18,1.52$ & 0.227 \\
\hline
\end{tabular}

Ref., reference category.

${ }^{*}$ The categories of $2-3$ cups/d and $\geq 4$ cups/d were collapsed into the single category of $\geq 2$ cups/d because of a small number of deaths from the specific causes in the category of $\geq 4$ cups/d.

†Age, sex, marital status, years of education, BMI, diabetes, smoking status, alcohol intake, vigorous exercise, use of any vitamin supplement, dietary intake (total energy, vegetables and fruits, red meat) and beverage consumption (black tea, green tea, Chinese tea, soda) were adjusted for.

Furthermore, considering dietary patterns which are potentially correlated with coffee consumption among Japanese adults $^{(24,25)}$, we additionally adjusted for intakes of fish, rice and dairy products. Statistical significance was defined as a two-sided $P$ value of less than 0.05. The statistical software package Stata SE version 14.2 was used for all analyses.

\section{Results}

Table 1 shows the baseline characteristics of all participants according to coffee consumption category. Compared with participants who did not drank coffee, those who drank two or more cups of coffee daily tended to be younger, male, married and current smokers. They also tended to have a higher level of education, consume a larger amount of alcohol, have higher intakes of total energy and red meat, and have a lower intake of vegetables and fruits. In addition, they were more likely to drink black tea, green tea, Chinese tea and soda, and were less likely to have diabetes and use any vitamin supplement. After stratification by sex, men and women showed the same tendency except alcohol consumption in men; that is, men who drank two or more cups of coffee daily tended to consume a smaller amount of alcohol (see online supplementary material, Supplemental Table S1).

During 16 years of follow-up (mean $=14 \cdot 1$ years), 5339 deaths were identified among 410352 total person-years. Table 2 shows the associations between coffee consumption and mortality from all causes, cancer, CVD and other causes among all participants and stratified by sex. Regarding all-cause mortality, coffee consumption was associated with reduced risk among all participants after adjusting for all potential confounders. Compared with the category of 'none', the HR (95\% CI) was $0.93(0.86$, $1.00)$ for $<1 \mathrm{cup} / \mathrm{d}, 0.84(0.76,0.93)$ for $1 \mathrm{cup} / \mathrm{d}, 0.81$ $(0.71,0.92)$ for $2-3 \mathrm{cups} / \mathrm{d}$ and $1.09(0.80,1.50)$ for $\geq 4$ cups/d ( $P$ for trend $<0.001$ ). A similar pattern was observed for men, but not for women.

Coffee consumption was not associated with mortality from cancer among all participants or women, although drinking 1 cup coffee/d was associated with reduced risk in men (Table 2). Regarding mortality from CVD, coffee consumption was associated with reduced risk among all participants. Compared with the category of 'none', the HR (95\% CI) were $0.87(0.77,0.99)$ for $<1 \mathrm{cup} / \mathrm{d}, 0.76$ $(0.63,0.92)$ for $1 \mathrm{cup} / \mathrm{d}, 0.67(0.50,0.89)$ for $2-3 \mathrm{cups} / \mathrm{d}$ and $1.39(0.77,2.49)$ for $\geq 4 \mathrm{cups} / \mathrm{d}$ ( $P$ for trend $=0.002)$. A similar pattern was observed for men and women, although inverse associations among women across all categories did not reach statistical significance. Specifically, coffee consumption was associated with reduced risk for mortality from stroke and IHD among all participants. The HR (95\% CI) for the categories of $<1 \mathrm{cup} / \mathrm{d}, 1 \mathrm{cup} /$ d, 2-3 cups/d and $\geq 4$ cups/d were $0.86(0.70,1.05), 0.64$ (0.47, 0.87), $0.65(0.41,1.02)$ and $1.11(0.41,3.06)$ for stroke mortality, and 0.68 (0.50, 0.91), 0.69 (0.46, 1.04), 0.50 (0.27, $0.92)$ and $1.41(0.50,3.98)$ for IHD mortality, respectively. Regarding mortality from other causes, coffee consumption was associated with reduced risk among all participants. 
Table 4 Sensitivity of hazard ratios (HR) for all-cause mortality among total participants, according to daily coffee consumption: Japanese adults aged $\geq 35$ years ( $n 29079$ ) followed from 1992 to 2008, Takayama study

\begin{tabular}{|c|c|c|c|c|c|c|c|c|c|c|}
\hline & \multicolumn{9}{|c|}{ Daily coffee consumption } & \multirow[b]{3}{*}{$P$ for trend } \\
\hline & \multirow[b]{2}{*}{ None } & \multicolumn{2}{|c|}{$<1$ cup } & \multicolumn{2}{|r|}{1 cup } & \multicolumn{2}{|c|}{ 2-3 cups } & \multicolumn{2}{|c|}{$\geq 4$ cups } & \\
\hline & & HR & $95 \% \mathrm{Cl}$ & HR & $95 \% \mathrm{Cl}$ & $\mathrm{HR}$ & $95 \% \mathrm{Cl}$ & $\mathrm{HR}$ & $95 \% \mathrm{Cl}$ & \\
\hline \multicolumn{11}{|l|}{$\begin{array}{l}\text { Excluding deaths within the first } \\
3 \text { years }(n 26319)\end{array}$} \\
\hline $\begin{array}{l}\text { Multivariate } \\
\text { Adjusting for the category of } \\
\text { smoking-years instead of smoking } \\
\text { status ( } n 25562 \text { ) }\end{array}$ & 1.00 (ref.) & 0.91 & $0.84,0.98$ & 0.84 & $0.75,0.93$ & 0.81 & $0.70,0.93$ & 1.03 & $0.73,1.45$ & 0.001 \\
\hline $\begin{array}{l}\text { Multivariate* } \\
\text { Adjusting for the category of } \\
\text { smoking-cessation years instead of } \\
\text { smoking status ( } n 25510)\end{array}$ & 1.00 (ref.) & 0.93 & $0.87,1.01$ & 0.85 & $0.76,0.94$ & 0.85 & $0.74,0.97$ & $1 \cdot 13$ & $0.74,0.97$ & 0.004 \\
\hline $\begin{array}{l}\text { Multivariate* } \\
\text { Additionally adjusting for the } \\
\text { categories of dietary intake } \\
\text { (fish, rice, dairy products; } n 26 \text { 943) }\end{array}$ & 1.00 (ref.) & 0.93 & $0.86,1.00$ & 0.83 & $0.75,0.92$ & 0.81 & $0.71,0.93$ & 1.04 & $0.71,0.93$ & $<0.001$ \\
\hline Multivariate* & 1.00 (ref.) & 0.93 & $0.86,1.00$ & 0.83 & $0.75,0.92$ & 0.80 & $0.70,0.92$ & 1.08 & $0.78,1.48$ & $<0.001$ \\
\hline
\end{tabular}

Ref., reference category.

*Age, sex, marital status, years of education, BMI, diabetes, smoking status, alcohol intake, vigorous exercise, use of any vitamin supplement, dietary intake (total energy, vegetables and fruits, red meat) and dairy beverage consumption (black tea, green tea, Chinese tea, soda) were adjusted for.

The HR $(95 \% \mathrm{CI})$ were $0.91(0.81,1.03)$ for $<1 \mathrm{cup} / \mathrm{d}, 0.85$ $(0.73,1.00)$ for $1 \mathrm{cup} / \mathrm{d}, 0.72(0.57,0.90)$ for $2-3 \mathrm{cups} / \mathrm{d}$ and $1.00(0.58,1.72)$ for $\geq 4 \mathrm{cups} / \mathrm{d}$ ( $P$ for trend $=0.005)$. A similar pattern was observed for men, but not for women.

Table 3 shows the associations between coffee consumption and mortality from other specific causes among all participants. In the multivariate models, coffee consumption was significantly associated with reduced risk of mortality from digestive and infectious diseases. Coffee consumption was also inversely associated with mortality from the other specific causes, although the point estimates were not statistically significant.

In the sensitivity analyses (Table 4), even after excluding deaths within the first 3 years, the main findings for all-cause mortality among all participants did not change substantially. Furthermore, adjusting for the categories of smoking-years or smoking-cessation years instead of smoking status also did not change the findings substantially.

\section{Discussion}

We used data from a population-based prospective cohort study in a Japanese city (the Takayama study) to examine the associations of coffee consumption with all-cause and cause-specific mortality after adjusting for important dietary and lifestyle factors. The results showed that drinking coffee, even $1 \mathrm{cup} / \mathrm{d}$, was associated with reduced risk of mortality from all causes, CVD and other causes among all participants, although the associations for $\geq 4 \mathrm{cups} / \mathrm{d}$ were no more significant. In the stratified analyses by sex, similar tendencies were observed for men, but not for women. Furthermore, coffee consumption was also inversely associated with mortality specifically from infectious and digestive diseases.

Although we found that drinking 1 cup coffee/d was associated with reduced risk for mortality from all and other specific causes, previous studies indicated that risks were lowest at 3-4 cups/d for all-cause and CVD mortality with no further reductions at $>4 \mathrm{cups} / \mathrm{d}^{(5,7,26)}$. In the present study, there were few participants who drank $\geq 4$ cups coffee/d ( $n$ 483, 1.7\%); therefore, we may have failed to observe a tendency in these associations.

The present finding of reduced risk for all-cause mortality is consistent with meta-analyses ${ }^{(4-7,26)}$ and recent large-scale cohort studies in Western countries (targeting multi-ethnic populations $)^{(11,12)}$ and Japan ${ }^{(16)}$. In the present study, the risks decreased by 16 and $19 \%$ for the categories of $1 \mathrm{cup} / \mathrm{d}$ and 2-3 cups/d, respectively, compared with the 'none' category. These findings are similar to those from large-scale cohort studies ( $15 \%$ for $1 \mathrm{cup} / \mathrm{d}$ and $20 \%$ for $2-3 \mathrm{cups} / \mathrm{d}$ in risk reductions among Japanese Americans compared with the 'none' category ${ }^{(11)}$, and $15 \%$ for the $1-2$ cups/d category and $24 \%$ for the 3-4 cups/d category in risk reductions compared with the 'almost-none' category $\left.{ }^{(16)}\right)$.

Coffee consumption was inversely associated with reduced risk for mortality from CVD, but not from cancer in the present study. This finding is consistent with three meta-analyses ${ }^{(5-7)}$ and previous studies in Japan ${ }^{(14-16,27)}$. The protective associations on mortality from CVD are plausible because of the presence of various bioactive compounds in coffee, as mentioned above. By contrast, the association of coffee with cancer mortality may vary by cancer site (e.g. probable protective associations between coffee consumption and liver and endometrial cancers are suggested $\left.{ }^{(3,28)}\right)$. Further studies evaluating 
coffee's associations with site-specific cancer mortality in non-Western countries are needed.

After stratification by sex, the inverse associations with mortality from all causes, CVD and other causes did not reach statistical significance in women. The potential reason is that women appeared to report coffee consumption less accurately than men. This may result in non-differential misclassifications, just making the estimates towards the null. It is also possible that caffeine, a coffee compound, could have sex-differentiated impacts on health ${ }^{(8,29)}$. For example, the CVD mortality risk in women is lower than that in men, which is influenced by the interactions between genetic, hormonal and environmental factors ${ }^{(30,31)}$. These factors could have sex-differentiated impacts on the associations between coffee consumption and CVD mortality.

In addition, coffee consumption seemed to be inversely associated with other specific-cause (non-CVD, noncancer) mortality, although we found significant inverse associations only for mortality from infectious and digestive diseases. The bioactive compounds in coffee (e.g. polyphenol, caffeine and melanoidins) could exert such beneficial effects on liver function and chronic inflammation ${ }^{(1,2)}$. Past studies reported that coffee consumption was associated with lower levels of liver enzymes, C-reactive protein and adiponectin ${ }^{(12,32-34)}$. Therefore, it is likely that coffee consumption is also inversely associated with mortality from respiratory disease and endocrine/metabolic disease, as suggested by previous studies ${ }^{(8,11,12,16,35,36)}$. The nonsignificant inverse associations we observed could be due to a small number of cases. Future studies evaluating associations between coffee consumption and specific causes of death are thus awaited.

The major strengths of the present study are its prospective cohort design with 16 years of follow-up, its high participation rate $(85.3 \%)$, use of a valid dietary questionnaire and considering important potential confounding factors simultaneously. Therefore, the present findings provide further evidence for the protective effects of coffee consumption among non-white, non-Western populations.

The present study has several limitations. First, it did not provide strong evidence for high coffee consumption, despite the dose-response relationships in the inverse associations between coffee consumption and all-cause mortality and mortality specifically from CVD, infectious and digestive diseases. Second, coffee consumption was assessed at baseline only and may not reflect long-term coffee consumption, although a study of three prospective US cohorts indicated that a single assessment can capture coffee drinking habits over $10-20$ years ${ }^{(36)}$. Indeed, participants with chronic disease may reduce their coffee consumption, but we excluded those participants from the analyses. Moreover, we conducted sensitivity analyses excluding deaths within the first 3 years, which did not change our findings substantially. However, the referent category of none could include those who had quit drinking coffee before baseline because of known or undiagnosed symptoms. Third, we did not collect information on how the participants prepared and served coffee, although its bioactive compounds vary depending on bean type, degree of roasting and preparation method, including quality of beans, grind settings and brewing methods ${ }^{(8,37)}$. Furthermore, we cannot differentiate between canned, instant or brewed coffee and cannot evaluate the associations of decaffeinated coffee because drinking decaffeinated coffee was likely to be rare in Japan ${ }^{(16)}$. These factors may result in moving the HR towards the null. Finally, we cannot rule out the possibility of residual confounding due to smoking status, which would be an important potential confounding factor. However, sensitivity analyses using two different smoking categories did not change the main findings substantially. In large-scale cohort studies, the results for the associations between coffee consumption and mortality were similar across smoking categories (i.e. never, former or current) ${ }^{(11,12,35)}$.

\section{Conclusion}

In conclusion, after adjusting for important dietary and lifestyle confounders, we demonstrated that drinking coffee, even $1 \mathrm{cup} / \mathrm{d}$, was associated with reduced risk of all-cause mortality and mortality specifically from CVD, infectious and digestive diseases. Thus, a little coffee consumption will contribute to increasing the health and long life of people in Asian countries, such as Japan.

\section{Acknowledgements}

Financial support: This work was supported in part by grants from the Ministry of Education, Culture, Sports, Science, and Technology and from the National Cancer Center Research and Development Fund, Japan. The sponsors were not involved in deciding the study design, the collection, analysis and interpretation of data, the writing of the report or the decision to submit this paper for publication. Conflict of interest: C.N. is an advisor to the All Japan Coffee Association. The other authors have no conflicts of interest to disclose. Authorship: M.Y., Y.G., F.M., S.K. and T.U. designed the study and analytical strategy; C.N. obtained data; K.W., M.Y. and C.N. performed analysis and interpretation of data; M.Y. drafted the initial manuscript; K.W., Y.G., F.M., S.K., T.U. and C.N. reviewed and revised the manuscript; C.N. obtained the grants and supervised the study; and all authors approved the final manuscript as submitted. Ethics of human subject participation: This study was approved by the Ethics Committee of Gifu University Graduate School of Medicine. 


\section{Supplementary material}

To view supplementary material for this article, please visit https://doi.org/10.1017/S1368980019000764

\section{References}

1. Nieber K (2017) The impact of coffee on health. Planta Med 83, 1256-1263.

2. Ludwig IA, Clifford MN, Lean MEJ et al. (2014) Coffee: biochemistry and potential impact on health. Food Funct 5, 1695-1717.

3. International Agency for Research on Cancer (2016) Q\&A on Monographs Volume 116: Coffee, maté, and very hot beverages. https://www.iarc.fr/wp-content/uploads/2018/07/ Monographs-QA_Vol116.pdf (accessed March 2019).

4. Je Y \& Giovannucci E (2014) Coffee consumption and total mortality: a meta-analysis of twenty prospective cohort studies. Br J Nutr 111, 1162-1173.

5. Crippa A, Discacciati A, Larsson SC et al. (2014) Coffee consumption and mortality from all causes, cardiovascular disease, and cancer: a dose-response meta-analysis. Am J Epidemiol 180, 763-775.

6. Malerba S, Turati F, Galeone C et al. (2013) A meta-analysis of prospective studies of coffee consumption and mortality for all causes, cancers and cardiovascular diseases. Eur J Epidemiol 28, 527-539.

7. Grosso G, Micek A, Godos J et al. (2016) Coffee consumption and risk of all-cause, cardiovascular, and cancer mortality in smokers and non-smokers: a dose-response meta-analysis. Eur J Epidemiol 31, 1191-1205.

8. Poole R, Kennedy OJ, Roderick P et al. (2017) Coffee consumption and health: umbrella review of meta-analyses of multiple health outcomes. BMJ 359, j5024.

9. Loftfield E, Freedman ND, Dodd KW et al. (2016) Coffee drinking is widespread in the United States, but usual intake varies by key demographic and lifestyle factors. J Nutr 146, $1762-1768$

10. Loftfield E, Freedman ND, Graubard BI et al. (2015) Association of coffee consumption with overall and causespecific mortality in a large US prospective cohort study. Am J Epidemiol 182, 1010-1022.

11. Park S-Y, Freedman ND, Haiman CA et al. (2017) Association of coffee consumption with total and cause-specific mortality among nonwhite populations. Ann Intern Med 167, 228-235.

12. Gunter MJ, Murphy N, Cross AJ et al. (2017) Coffee drinking and mortality in 10 European countries: a multinational cohort study. Ann Intern Med 167, 236-247.

13. Iwai N, Ohshiro H, Kurozawa Y et al. (2002) Relationship between coffee and green tea consumption and all-cause mortality in a cohort of a rural Japanese population. J Epidemiol 12, 191-198.

14. Sugiyama K, Kuriyama S, Akhter M et al. (2010) Coffee consumption and mortality due to all causes, cardiovascular disease, and cancer in Japanese women. J Nutr 140, 1007-1013.

15. Tamakoshi A, Lin Y, Kawado M et al. (2011) Effect of coffee consumption on all-cause and total cancer mortality: findings from the JACC study. Eur J Epidemiol 26, 285-293.

16. Saito E, Inoue M, Sawada N et al. (2015) Association of coffee intake with total and cause-specific mortality in a Japanese population: the Japan Public Health Center-based Prospective Study. Am J Clin Nutr 101, 1029-1037.

17. All Japan Coffee Association (2017) Statistics: supply and demand of coffee in Japan (in Japanese). http://coffee. ajca.or.jp/data (accessed August 2018).
18. Chei C-L, Loh JK, Soh A et al. (2018) Coffee, tea, caffeine, and risk of hypertension: the Singapore Chinese Health Study. Eur J Nutr 57, 1333-1342.

19. Kim K, Kim K \& Park SM (2016) Association between the prevalence of metabolic syndrome and the level of coffee consumption among Korean women. PLoS One 11, $\mathrm{e} 0167007$.

20. Shimizu H (1996) The Basic Report on Takayama Study. Gifu: Gifu University.

21. Nagata C, Takatsuka N, Shimizu N et al. (2004) Sodium intake and risk of death from stroke in Japanese men and women. Stroke 35, 1543-1547.

22. Shimizu H, Ohwaki A, Kurisu Y et al. (1999) Validity and reproducibility of a quantitative food frequency questionnaire for a cohort study in Japan. Jpn J Clin Oncol 29, 38-44.

23. Willett W (editor) (2012) Implications of total energy intake for epidemiologic analyses. In Nutritional Epidemiology, 3rd ed., pp. 260-286. New York: Oxford University Press.

24. Maruyama K, Iso H, Date C et al. (2013) Dietary patterns and risk of cardiovascular deaths among middle-aged Japanese: JACC Study. Nutr Metab Cardiovasc Dis 23, 519-527.

25. Shimazu T, Kuriyama S, Hozawa A et al. (2007) Dietary patterns and cardiovascular disease mortality in Japan: a prospective cohort study. Int J Epidemiol 36, 600-609.

26. Grosso G, Stepaniak U, Micek A et al. (2017) Coffee consumption and mortality in three Eastern European countries: results from the HAPIEE (Health, Alcohol and Psychosocial factors in Eastern Europe) study. Public Health Nutr 20, 82-91.

27. Mineharu Y, Koizumi A, Wada Y et al. (2011) Coffee, green tea, black tea and oolong tea consumption and risk of mortality from cardiovascular disease in Japanese men and women. J Epidemiol Community Health 65, 230-240.

28. World Cancer Research Fund \& American Institute for Cancer Research (2018) Non-alcoholic drinks and the risk of cancer. In Diet, Nutrition, Physical Activity and Cancer: A Global Perspective. Continuous Update Project Expert Report 2018, pp. 1-62. London: WCRF International.

29. Temple JL \& Ziegler AM (2011) Gender differences in subjective and physiological responses to caffeine and the role of steroid hormones. J Caffeine Res 1, 41-48.

30. Winham SJ, de Andrade M \& Miller VM (2014) Genetics of cardiovascular disease: importance of sex and ethnicity. Atherosclerosis 241, 219-228.

31. Deo R \& Albert CM (2012) Epidemiology and genetics of sudden cardiac death. Circulation 125, 620-637.

32. Xiao Q, Sinha R, Graubard BI et al. (2014) Inverse associations of total and decaffeinated coffee with liver enzyme levels in National Health and Nutrition Examination Survey 1999-2010. Hepatology 60, 2091-2098.

33. Zhang Y \& Zhang D-Z (2018) Is coffee consumption associated with a lower level of serum C-reactive protein? A meta-analysis of observational studies. Int J Food Sci Nutr 69, 985-994

34. Zhang Y \& Zhang D-Z (2018) Associations of coffee consumption with circulating level of adiponectin and leptin. A meta-analysis of observational studies. Int J Food Sci Nutr 69, 1003-1012.

35. Freedman ND, Park Y, Abnet CC et al. (2012) Association of coffee drinking with total and cause-specific mortality. NEngl J Med 366, 1891-904.

36. Ding M, Satija A, Bhupathiraju SN et al. (2015) Association of coffee consumption with total and cause-specific mortality in 3 large prospective cohorts. Circulation 132, 2305-2315.

37. Corrêa TAF, Monteiro MP, Mendes TMN et al. (2012) Medium light and medium roast paper-filtered coffee increased antioxidant capacity in healthy volunteers: results of a randomized trial. Plant Foods Hum Nutr 67, 277-282. 